\title{
The aetiology and trajectory of anabolic- androgenic steroid use initiation: a systematic review and synthesis of qualitative research
}

\author{
Dominic Sagoe ${ }^{1 *}$, Cecilie Schou Andreassen ${ }^{1,2}$ and Ståle Pallesen ${ }^{1}$
}

\begin{abstract}
Background: To our knowledge, there has never been a systematic review and synthesis of the qualitative literature on the trajectory and aetiology of nonmedical anabolic-androgenic steroid (AAS) use.

Methods: We systematically reviewed and synthesized qualitative literature gathered from searches in PsycINFO, PubMed, ISI Web of Science, Google Scholar, and reference lists of relevant literature to investigate AAS users' ages of first use and source(s), history prior to use, and motives/drives for initiating use. We adhered to the recommendations of the UK Economic and Social Research Council's qualitative research synthesis manual and the PRISMA guidelines.

Results: A total of 44 studies published between 1980 and 2014 were included in the synthesis. Studies originated from 11 countries: the United States $(n=18)$, England $(n=8)$, Australia $(n=4)$, Sweden $(n=4)$, both England and Wales $(n=2)$, and Scotland $(n=2)$. One study each originated from Brazil, Bulgaria, Canada, France, Great Britain, and Norway. The majority of AAS users initiated use before age 30. Sports participation (particularly power sports), negative body image, and psychological disorders such as depression preceded initiation of AAS use for most users. Sources of first AAS were mainly users' immediate social networks and the illicit market. Enhanced sports performance, appearance, and muscle/strength were the paramount motives for AAS use initiation.
\end{abstract}

Conclusions: Our findings elucidate the significance of psychosocial factors in AAS use initiation. The proliferation of AAS on the illicit market and social networks demands better ways of dealing with the global public health problem of AAS use.

Keywords: Anabolic-androgenic steroids, Metasynthesis, Narrative synthesis, Systematic review, Aetiology, Trajectory, Qualitative research, Interview

\section{Background}

Several qualitative investigations have sought to understand the aetiology and trajectory of nonmedical AAS use initiation. However, to our knowledge, there has never been a systematic review and synthesis of the qualitative literature on this important area of nonmedical AAS use. An investigation of this type is important because a global perspective of nonmedical AAS use initiation is necessary for the understanding of this global public health problem [1].

A review and synthesis of the qualitative research on AAS use initiation is also important in light of expressed

\footnotetext{
* Correspondence: dominic.sagoe@psysp.uib.no

'Department of Psychosocial Science, University of Bergen, Christiesgate 12, 5015 Bergen, Norway

Full list of author information is available at the end of the article
}

concern regarding the validity and reliability of survey research on AAS use [2]. Moreover, it has been suggested that the failure of health practitioners and public health officials to appreciate people's perception of antecedents and risk factors is a major hindrance to the success of public health interventions $[3,4]$. Hence, data on initiation and trajectories of AAS use are important for prevention purposes.

We carried out, as far as we are aware, the pioneering systematic review and synthesis of the qualitative studies presenting data on the initiation of nonmedical AAS use. The United Kingdom's Economic and Social Research Council's manual on the synthesis of qualitative literature [5] indorses the formulation of research questions or hypothesis prior to synthesis. The research questions guiding 
the present study were: (a) at what age(s) do AAS users have their debut?, (b) what are the psychosocial histories of AAS users prior to the initiation of AAS use?, (c) what are the sources of AAS users' first AAS?, and (d) what are the motives and drives for initiating AAS use?

\section{Method}

\section{Search strategy and inclusion criteria}

We conducted a comprehensive literature search in PsycINFO, PubMed, ISI Web of Science, and Google Scholar. The following keywords: 'anabolic steroid', 'doping', and 'performance enhancing drug', were each used in combination with 'interview', 'focus group', and 'qualitative' for searches in PubMed and ISI Web of Science. Due to unusually high superfluous returns from the above permutation of keywords, 'anabolic steroid + doping + performance enhancing drug + interview + focus group + qualitative' was used in searches in PsycINFO and Google Scholar. The literature search was completed in June 2014. From an initial pool of 10,106 hits, 7,720 articles were evaluated after removing duplicates. In addition, a manual check of reference lists of identified studies was conducted in search of potential unidentified studies. Searches were also conducted in online databases and websites. We identified 4 new articles through this grey literature search. Thus, a total of 7,724 were settled on after eliminating duplicates. After evaluating the 7,724 papers based on titles and abstracts, 95 full-text papers were retrieved for screening.

After initial screening of the 95 full-text papers, 68 papers were identified. Of the 68 papers scrutinized, 35 studies met the following key criteria for inclusion: (a) studies presented original information on the experiences of AAS users (b) studies employed qualitative approaches in data collection (interviews, focus groups, or case studies) and presentation of results, and (c) studies were published in English. Four recent studies [6-9] and five others [10-14] were later discovered and included in the analysis. We again inspected the characteristics of extracted studies for similarities to curb duplicate extraction and synthesis. Thus, a total of 44 articles were included in the analysis. The literature search strategy adhered to Shaw et al.'s [15] recommendations for finding qualitative research as well as the Preferred Reporting Items for Systematic Reviews and Meta-Analyses (PRISMA) guidelines [16]. Figure 1 presents the process of the search and selection of relevant studies according to the PRISMA guidelines.

\section{Data extraction and synthesis}

The first author scrutinized and selected studies. Smith et al.'s [17] Interpretative Phenomenological Analysis

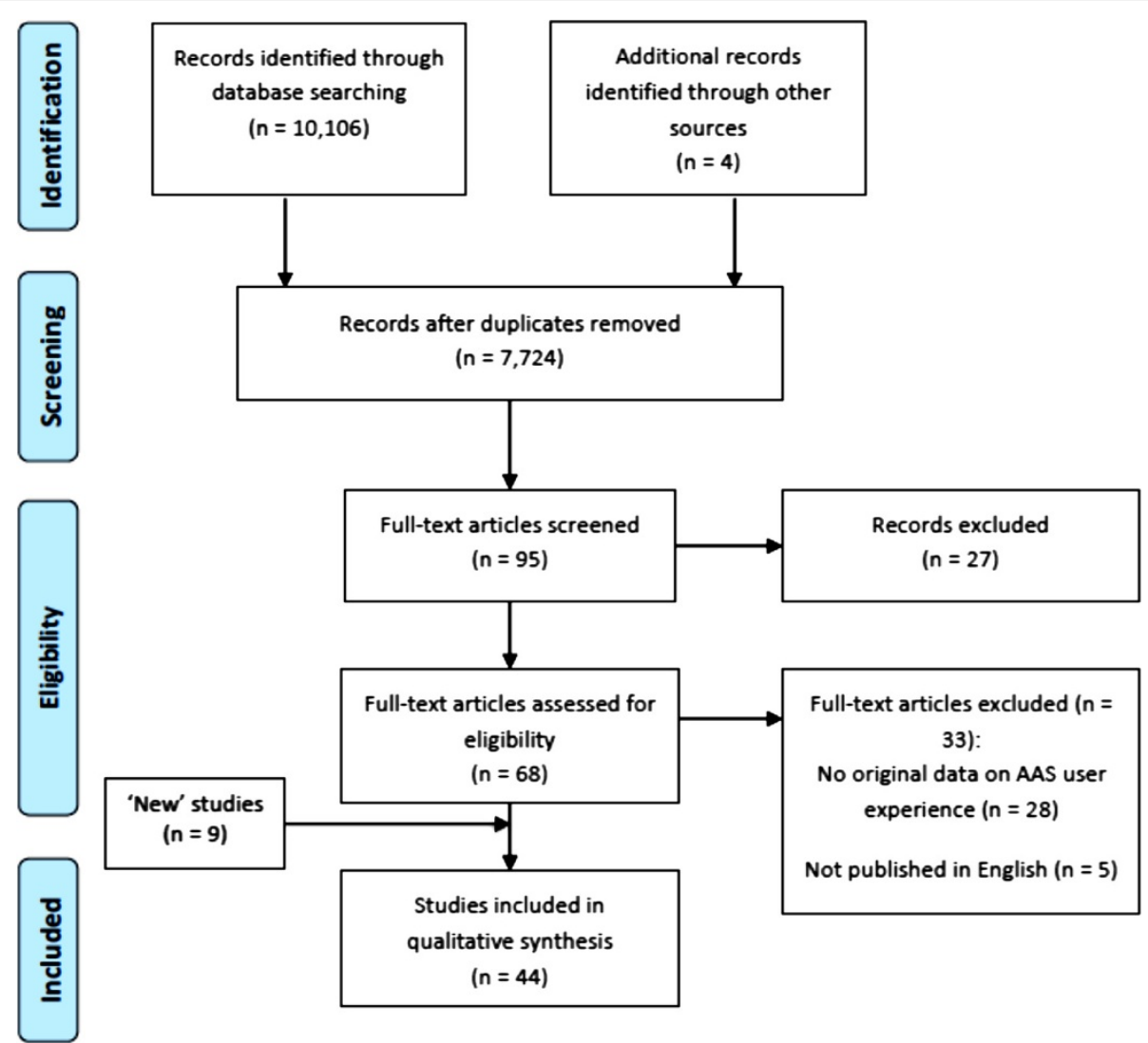

Figure 1 Flow diagram of systematic literature search. 
(IPA) was used to analyse the studies because it facilitates in-depth exploration of the meanings of experiences [18]. Each full-text paper was regarded as a transcript. The first author read through the full-text papers several times, gaining an overall sense of the themes in the studies through this process. These themes were then highlighted. We developed a standardized data extraction form unto which the first author and another reviewer independently extracted the following data from the included studies: author name and publication year, country, study type, type of AAS users involved in the study, and recruitment site or mode. These characteristics are presented in Table 1. The first author independently coded the full-text papers according to the presence or absence of the following themes: (a) age(s) of first use, (b) history prior to use, (c) source(s) of first AAS, and (d) motive(s)/drive(s) for initiating use. These characteristics are presented in Table 2. Statistical inferences have little meaning in qualitative synthesis. However, the presence of a theme in multiple studies may be evidence of the validity of the theme [4]. In this regard, we have presented all the studies that fall under each theme.

\section{Quality of extraction, included studies, and synthesis}

To assess the quality of the extraction, we calculated inter-reviewer reliability for the two reviewers in SPSS version 20 (IBM Corp.) [54]. Sensitivity analysis is conducted in the synthesis of qualitative research to examine the effect of the exclusion of high or poor quality studies on the overall findings. We assessed the relevance of the included papers according to the four themes: (a) age(s) of first use, (b) history prior to use, (c) source(s) of first AAS, and (d) motivation(s) for use (see Table 2). Each theme was scored ' 1 ' thus yielding a possible total score of ' 4 '. Subsequently, we excluded studies that scored $\leq 2$ out of 4 on the themes and investigated the effect of the exclusion on our synthesis and results. Moreover, as most of the included studies were conducted in the United States, we excluded the United States studies to investigate the effect of the exclusion on the quality of our synthesis and results.

\section{Results and discussion}

\section{Strength of extraction, included studies, and synthesis}

The inter-reviewer reliability for the reviewers was found to be Kappa $=0.82(p<0.001)$ indicating very good agreement between the two reviewers [55]. Consensus was reached on discrepant extractions through further review and discussion. Thirty-eight (38) of the 44 studies scored $\geq 3$ out of 4 on the themes and were thus deemed to be of high relevance. Six studies [31,36,37,41,46,52] scored $\leq 2$ out of 4 on relevance and were therefore excluded in the quality analysis. However, when we removed the study characteristics generated from these studies in the sensitivity analysis, our themes or results did not change. Consequently, we retained them in the final analysis. Similarly, the removal of the study characteristics generated from the studies originating from the United States did not affect the quality of our themes or results. Thus, they were also retained in the final analysis. The sensitivity analysis therefore indicated a strong synthesis of included studies.

\section{Description of studies}

A total of 44 studies were included in the metasynthesis. Participants' ages ranged from 14 to 63 years. The year of publication of the studies ranged from 1980 [19] to 2014 $[6-9,11]$. Studies originated from 11 countries although most originated from the United States $(n=18)$, followed by England $(n=8)$, Australia $(n=4)$, Sweden $(n=4)$, both England and Wales $(n=2)$, and Scotland $(n=2)$. Moreover, one study each originated from Brazil, Bulgaria, Canada, France, Great Britain, and Norway. Twenty-nine studies [6-8,12,19-21,25-27,30-36,38-47,49,51,52] used interviews, six were case studies [14,22,28,29,45,53], one used interviews and focus groups [9], and eight [10,11, $13,23,24,37,48,50]$ used interviews supported by a questionnaire. For the eight studies that used both interviews and questionnaires, we relied on the qualitative results generated from the interviews.

\section{Narrative synthesis}

We found that majority of studies had participants initiating use before they were 30 years old. In addition, histories of negative body image, psychological disorders such as mood and depressive disorders, and participation in power sports preceded initiation of AAS use for most persons. We also found that sources of first AAS were mainly users' immediate social networks and the illicit market. Furthermore, we found that motives for AAS use were mainly enhanced sports performance, appearance, and muscle or strength.

\section{Age of AAS use initiation}

Of the 24 studies that presented the ages at which participants initiated AAS use, initiation ages ranged from 14 to 54 years. However, only 5 of the 24 studies presented participants that initiated AAS use after age 30 consistent with evidence that about $80 \%$ of AAS users initiate use before age 30 [56]. It must be noted that some studies did not specify the ages at which some or all respondents initiated AAS use (See Table 3).

\section{Pre-initiation history}

Prior to initiating AAS use, participants had diverse backgrounds including sports (particularly power sports) participation, maladaptive relationships, psychopathology, 
Table 1 Qualitative studies presenting data on AAS use initiation

\begin{tabular}{|c|c|c|c|c|}
\hline $\begin{array}{l}\text { First author, year, } \\
\text { reference }\end{array}$ & Country/countries & Study type & AAS user(s) & Recruitment site(s)/mode \\
\hline Annitto 1980 [19] & USA & Interview & 17-year-old male bodybuilder & Clinic \\
\hline Bardick 2006 [20] & Canada & Interview & 8 male weightlifters aged 21 to 35 years & Gymnasium \\
\hline Bilard 2011 [21] & France & Interview & 203 bodybuilders & Voluntary \\
\hline Boyadjiev 2000 [22] & Bulgaria & Case study & 20-year-old male bodybuilder & Clinic \\
\hline $\begin{array}{l}\text { Copeland } 2000[23] \text { and } \\
\text { Peters } 1997[24]\end{array}$ & Australia & $\begin{array}{l}\text { Interview and } \\
\text { questionnaire }^{\dagger}\end{array}$ & $\begin{array}{l}100 \text { persons ( } 6 \text { female) } \\
\text { aged } 18 \text { to } 50 \text { years }\end{array}$ & $\begin{array}{l}\text { Gymnasium, sports shops and } \\
\text { associations, syringe exchange } \\
\text { centre, radio interviews, } \\
\text { advertisements }\end{array}$ \\
\hline Cornford 2014 [9] & England & $\begin{array}{l}\text { Interview and } \\
\text { focus group }\end{array}$ & 30 males aged 20 to 40 years & Syringe exchange centre \\
\hline Fudala 2003 [25] & USA & Interview & 7 males aged 22 to 33 years & Gymnasium and community \\
\hline Grogan 2006 [26] & England & Interview & $\begin{array}{l}11 \text { bodybuilders ( } 6 \text { female) } \\
\text { aged } 20 \text { to } 39 \text { years }\end{array}$ & Gymnasium \\
\hline Gruber 1999 [27] & USA & Interview & 5 female bodybuilders & Gymnasium \\
\hline Hegazy 2013 [28] & USA & Case study & 28-year-old male & Clinic \\
\hline Joubert 2014 [7] & England & Interview & 6 males aged 26 to 42 years & Addiction charity \\
\hline Katz 1990 [29] & USA & Case study & 23-year-old male bodybuilder & Gymnasium \\
\hline Khorrami 2002 [30] & USA & Interview & $\begin{array}{l}2 \text { male weightlifters aged } \\
24 \text { and } 29 \text { years }\end{array}$ & Voluntary \\
\hline Kimergård $2014[6,8]$ & England and Wales & Interview & $\begin{array}{l}24 \text { males aged } 21 \text { to } 61 \text { years; } \\
\text { mean age } 34 \text { years }\end{array}$ & $\begin{array}{l}\text { Gymnasium, prison, steroid } \\
\text { clinic and charity, syringe } \\
\text { exchange centre }\end{array}$ \\
\hline Klötz 2010 [31] & Sweden & Interview & 33 male prisoners aged 21 to 52 years & Prison \\
\hline Korkia 1993 [12] & $\begin{array}{l}\text { England, Scotland, } \\
\text { and Wales }\end{array}$ & Interview & $\begin{array}{l}110 \text { persons ( } 13 \text { female) } \\
\text { aged } 16 \text { to } 63 \text { years }\end{array}$ & $\begin{array}{l}\text { Gymnasium, clinic, syringe } \\
\text { exchange centre }\end{array}$ \\
\hline Korkia 1996 [13] & England & $\begin{array}{l}\text { Interview and } \\
\text { questionnaire }^{+}\end{array}$ & 15 females; mean age 28 years & Not specified \\
\hline Kusserow 1990 [32] & USA & Interview & $\begin{array}{l}72 \text { ( } 6 \text { female) persons (mostly adolescents); } \\
14 \text { to } 25 \text { years; mean age } 20 \text { years }\end{array}$ & Not specified \\
\hline Malone 1995 [33] & USA & Interview & $\begin{array}{l}77 \text { (6 female) powerlifters and } \\
\text { bodybuilders }\end{array}$ & Gymnasium \\
\hline $\begin{array}{l}\text { Maycock } 2005 \text { [34], } 2007 \\
{[35]}\end{array}$ & Australia & Interview & 42 males & $\begin{array}{l}\text { Gymnasium, night club, } \\
\text { community }\end{array}$ \\
\hline McKillop 1987 [36] & Scotland & Interview & $\begin{array}{l}8 \text { male bodybuilders aged } \\
17 \text { to } 32 \text { years }\end{array}$ & Gymnasium \\
\hline Midgley 1999 [37] & England & $\begin{array}{l}\text { Interview and } \\
\text { questionnaire }^{\dagger}\end{array}$ & $\begin{array}{l}50 \text { male bodybuilders and } \\
\text { weight trainers aged } 17 \text { to } 46 \text { years }\end{array}$ & $\begin{array}{l}\text { Gymnasium and syringe } \\
\text { exchange centre }\end{array}$ \\
\hline Nøkleby 2013 [38] & Norway & Interview & 9 male drug users aged 22 to 35 years & Clinic \\
\hline O'Sullivan 2000 [39] & Australia & Interview & 41 males aged 16 to 36 years & Clinic \\
\hline Olrich 1999 [40] & USA & Interview & $\begin{array}{l}10 \text { male weightlifters; } 9 \text { aged } \\
18 \text { to } 35 \text { years, } 1 \text { aged } 57 \text { years }\end{array}$ & Gymnasium \\
\hline Pappa 2012 [41] & England & Interview & 9 athletes aged 19 to 26 years & $\begin{array}{l}\text { Community via snowball } \\
\text { sampling }\end{array}$ \\
\hline Petrocelli 2008 [42] & USA & Interview & 37 male gym users aged 19 to 43 years & Gymnasium \\
\hline Pope 1990 [43] & USA & Interview & $\begin{array}{l}3 \text { male arrested weightlifters } \\
\text { aged } 23,24 \text {, and } 32 \text { years }\end{array}$ & Justice system \\
\hline Pope 1993 [44] & USA & Interview & $\begin{array}{l}55 \text { bodybuilders; mean age } 28 \text { years; } 3 \\
\text { bodybuilders; } 19 \text { years, } 26 \text { years, } 27 \text { years }\end{array}$ & Gymnasium \\
\hline Pope 1996 [45] & USA & Case study & 16-year-old male & Clinic \\
\hline Pope 1996 [45] & USA & Interview & 9 male prisoners & Prison \\
\hline
\end{tabular}


Table 1 Qualitative studies presenting data on AAS use initiation (Continued)

\begin{tabular}{|c|c|c|c|c|}
\hline Rashid 2000 [14] & USA & Case study & 40-year-old male & Clinic \\
\hline Schwingel $2012[46]$ & Brazil & Interview & $\begin{array}{l}147 \text { male power sportspeople } \\
\text { aged } 18 \text { to } 42 \text { years }\end{array}$ & Exercise laboratory \\
\hline Scull 2013 [47] & USA & Interview & 7 male strippers & Strip club \\
\hline Skårberg 2007 [48] & Sweden & $\begin{array}{l}\text { Interview and } \\
\text { questionnaire }^{\dagger}\end{array}$ & 18 male drug users; mean age 35 years & Clinic \\
\hline Skårberg 2008 [49] & Sweden & Interview & 6 drug users (2 female) & Clinic \\
\hline $\begin{array}{l}\text { Skårberg } 2009 \text { [50] and } \\
2007 \text { [48] }\end{array}$ & Sweden & $\begin{array}{l}\text { Interview and } \\
\text { questionnaire }^{\dagger}\end{array}$ & $\begin{array}{l}32 \text { male drug users } 18 \text { male drug users; mean } \\
\text { age } 35 \text { years }\end{array}$ & Clinic \\
\hline Tallon 2007 [11] & Scotland & $\begin{array}{l}\text { Interview and } \\
\text { questionnaire }^{\dagger}\end{array}$ & $\begin{array}{l}30 \text { males aged } 18 \text { to } 43 \text { years; mean age } \\
27 \text { years }\end{array}$ & Gymnasium \\
\hline Todd $1987[51]$ & USA & Interview & $\begin{array}{l}2 \text { persons (27-year-old female weightlifter; } 1 \\
\text { former male NFL player) }\end{array}$ & Not specified \\
\hline Vassalo $2010[52]$ & USA & Interview & 39 male athletes aged 18 to 35 years & Acquaintances \\
\hline Walker 2011 [10] & England & $\begin{array}{l}\text { Interview and } \\
\text { questionnaire }^{\dagger}\end{array}$ & 41 males; 20 to 30 years (majority) & Syringe exchange centre \\
\hline Wilson-Fearon 1999 [53] & England & Case study & 29-year-old bodybuilder & Not specified \\
\hline
\end{tabular}

${ }^{+}$We relied on the qualitative results generated from the interview.

negative self and body image, deviant behaviour, and abuse of other drugs (See Table 4).

The most prominent feature of AAS users prior to initiation of use was participation in power sports such as bodybuilding, powerlifting, and weightlifting. This emerged in 23 studies [11-14,17,18,21,22,24-27,32,34-37,41,43,45,50, 52,55]. It emerged in Maycock and Howat's study [34] that users:

...had been weight training for three years prior to initiating anabolic steroid use. However, 11 of the interviewed subjects initiated use within one year of starting weight training (p. 319).

Similarly, participation in other sports such as athletics, cycling, hockey, and football emerged as a prominent feature of AAS users backgrounds prior to initiation of AAS use $[7,11,22,25,30,32,38,41,49,52]$. This is exemplified by Josh in Bardick et al.'s study [20]. Josh was a hockey player who "needed to take steroids to become the best" (p. 138). Similarly, Maycock and Howat [34] highlighted association with 'complacent' trainers or coaches as a feature of AAS users prior to the initiation of AAS use (p. 319).

Also, Gruber and Pope [27] recount the story of Ms. A. who "took all of the supplements and ergogenic drugs that her trainer recommended, including large doses of anabolic steroids". In Maycock and Howat's study [34]:

Four of the interviewed sample indicated that complacency by trainers and coaches contributed to their decision to consider use. The failure of coaches and officials to investigate large increases in body mass and strength achieved by other competitors contributed to their decision to explore use (p. 319).

AAS users also showed psychological syndromes such as mood and depressive disorders as well as troubled psychosocial histories including divorce, having suffered rape, poor parental connectedness or involvement, and poor social support $[14,25,27,28,44,45,48-50]$ prior to the initiation of AAS use. In one study [27], five females initiated AAS use after the experience of rape:

None used such drugs previously...Indeed, prior to experiencing rape, these five women believed that taking anabolic substances was a weakness...

Subsequent to their rape, they justified the decision to start using anabolic substances as being necessary to gain muscle mass and strength, because they thought it was impossible to grow big or strong enough "naturally" (p. 275).

Also evident as a feature of AAS users prior to initiation of AAS use was eating disorders such as anorexia nervosa $[25,44]$. Pope et al. [44] present the cases of four persons who initiated AAS use due to anorexia nervosa and reverse anorexia nervosa. Negative body image as well as low self-esteem and low self-efficacy also emerged as features of AAS users prior to the initiation of AAS use $[7,10,25,30,32,34,35,42,44]$. Cases 01 and 02 of Fudala et al.'s study [25] recount the stories of a male who "stated that he was using AASs because he lacked self-esteem and was not good-looking." and another who initiated AAS use because he "felt small and [needed to] become more muscular to accomplish [his] goals" (p. 123). 
Table 2 Characteristics of qualitative studies presenting data on AAS use initiation

\begin{tabular}{|c|c|c|c|c|}
\hline $\begin{array}{l}\text { First author, year, } \\
\text { reference }\end{array}$ & Initiation age(s) & History prior to use & Source(s) & Motive(s)/drive(s) for use \\
\hline Annitto 1980 [19] & 16 years & Weightlifting & Illicit market & Appearance \\
\hline Bardick 2006 [20] & Not specified & Weight training & Not specified & $\begin{array}{l}\text { Appearance, confidence, media, } \\
\text { personal security, psychological } \\
\text { well-being, sports }\end{array}$ \\
\hline Bilard 2011 [21] & Not specified & Bodybuilding & $\begin{array}{l}\text { Friends, dealers, others, } \\
\text { relatives, teammates }\end{array}$ & $\begin{array}{l}\text { Appearance, muscle, physiological } \\
\text { recovery, psychological, sports, } \\
\text { sports norm, other }\end{array}$ \\
\hline Boyadjiev 2000 [22] & 19 years & Bodybuilding, cycling & Not specified & Sports \\
\hline $\begin{array}{l}\text { Copeland } 2000[23] \\
\text { and Peters } 1997 \text { [24] }\end{array}$ & $\begin{array}{l}14 \text { to } 46 \text { years; } \\
\text { mean } 25 \text { years }\end{array}$ & Not specified & $\begin{array}{l}\text { Coaches/trainers, dealers, } \\
\text { doctors, friends, gym } \\
\text { employees, other, } \\
\text { pharmacists, mail order } \\
\text { relatives, veterinarians }\end{array}$ & $\begin{array}{l}\text { Appearance, muscle, other, } \\
\text { physiological recovery/injury } \\
\text { prevention, sports }\end{array}$ \\
\hline Cornford 2014 [9] & $\leq 30$ years $(n=14)$ & Not specified & Not specified & $\begin{array}{l}\text { Muscle, personal security, } \\
\text { physiological recovery, sports }\end{array}$ \\
\hline Fudala 2003 [25] & $\leq 26$ years & $\begin{array}{l}\text { Negative body image, } \\
\text { poor self-esteem, psy- } \\
\text { chological disorders, } \\
\text { troubled background }\end{array}$ & Not specified & Appearance, psychological \\
\hline Fudala 2003 [25] & $\leq 31$ years & $\begin{array}{l}\text { Negative body image, } \\
\text { low self-efficacy, trou- } \\
\text { bled background }\end{array}$ & Not specified & Appearance, muscle \\
\hline Fudala 2003 [25] & 17 years & Football & Relative & Appearance, muscle, sports \\
\hline Fudala 2003 [25] & 26 years & Troubled background & Not specified & Appearance, muscle \\
\hline Fudala 2003 [25] & 21 years & $\begin{array}{l}\text { Binge eating, } \\
\text { psychological } \\
\text { disorders, troubled } \\
\text { background }\end{array}$ & Not specified & Not specified \\
\hline Fudala 2003 [25] & 27 years & $\begin{array}{l}\text { Troubled background, } \\
\text { weightlifting }\end{array}$ & Friend & Sports \\
\hline Fudala 2003 [25] & 24 years & Bodybuilding & Not specified & Sports \\
\hline Grogan 2006 [26] & $\begin{array}{l}15 \text { years, } 16 \text { years, } \\
18 \text { years, } 19 \text { years, } \\
20 \text { years, } 21 \text { years, } \\
23 \text { years, } 29 \text { years }\end{array}$ & Bodybuilding & Not specified & $\begin{array}{l}\text { Appearance, media, occupational, } \\
\text { sports, sport/social norm }\end{array}$ \\
\hline Gruber 1999 [27] & Not specified & $\begin{array}{l}\text { Polydrug use, } \\
\text { psychological } \\
\text { disorders, troubled } \\
\text { background }\end{array}$ & Trainer & Appearance, muscle, personal security \\
\hline Hegazy 2013 [28] & 22 years & $\begin{array}{l}\text { Polydrug use, } \\
\text { psychological } \\
\text { disorders, troubled } \\
\text { background }\end{array}$ & Friends & Appearance, muscle, recovery \\
\hline Joubert 2014 [7] & 16 to 24 years & $\begin{array}{l}\text { Low self-esteem, } \\
\text { negative body image, } \\
\text { troubled background }\end{array}$ & Not specified & $\begin{array}{l}\text { Appearance, confidence, family influence, } \\
\text { muscle, peer influence, personal security, } \\
\text { psychological well-being, self-esteem, } \\
\text { social pressure }\end{array}$ \\
\hline Katz 1990 [29] & 21 years & Bodybuilding & Not specified & Sports \\
\hline Khorrami 2002 [30] & Not specified & $\begin{array}{l}\text { Football, negative } \\
\text { body image, } \\
\text { weightlifting }\end{array}$ & Gym employee & Appearance, family influence, muscle, sports \\
\hline Kimergård 2014 [6,8] & $\begin{array}{l}16 \text { years; mean } \\
\text { age } 25 \text { years }\end{array}$ & Not specified & Not specified & Appearance, muscle, occupational, sports \\
\hline Klötz 2010 [31] & Not specified & Not specified & Not specified & $\begin{array}{l}\text { Aggression, appearance, muscle, other, } \\
\text { psychological, sports, sport/social norm }\end{array}$ \\
\hline Korkia 1993 [12] & & Weight training & & Muscle, physiological recovery, sports \\
\hline
\end{tabular}


Table 2 Characteristics of qualitative studies presenting data on AAS use initiation (Continued)

\begin{tabular}{|c|c|c|c|c|}
\hline & $\begin{array}{l}16 \text { years, } 18 \text { years, } \\
32 \text { years, } 54 \text { years }\end{array}$ & & $\begin{array}{l}\text { Coach, dealers, doctors, } \\
\text { friends/teammates, gym } \\
\text { owner/employee }\end{array}$ & \\
\hline Korkia 1996 [13] & 19 years, 23 years & Not specified & $\begin{array}{l}\text { Friends, gym owners/ } \\
\text { employees, husbands/ } \\
\text { boyfriends }\end{array}$ & Muscle, sports \\
\hline Kusserow 1990 [32] & $\begin{array}{l}14 \text { years, } 15 \text { years, } \\
17 \text { years, } 18 \text { years, } \\
\leq 25 \text { years }^{+}\end{array}$ & $\begin{array}{l}\text { Football, } \\
\text { bodybuilding, } \\
\text { negative body image, } \\
\text { polydrug use }\end{array}$ & $\begin{array}{l}\text { Coach/team doctor, dealers, } \\
\text { doctors, friends/teammates, } \\
\text { gym employees, pharmacists, } \\
\text { veterinarians }\end{array}$ & $\begin{array}{l}\text { Aggression, sports scholarship, appearance, } \\
\text { coaches' approval, famous athletes, media } \\
\text { influence, parental approval, peer influence, } \\
\text { sexual attraction, sports }\end{array}$ \\
\hline Malone 1995 [33] & 24 years & Weightlifting & Not specified & $\begin{array}{l}\text { Appearance, muscle, injury prevention/recovery, } \\
\text { sports, sport norm }\end{array}$ \\
\hline $\begin{array}{l}\text { Maycock } 2005[34] \\
\text { and } 2007[35]\end{array}$ & 24 years, 25 years & $\begin{array}{l}\text { Complacent trainers, } \\
\text { negative body image, } \\
\text { weight training }\end{array}$ & Dealers & $\begin{array}{l}\text { Appearance, aggression, coaches' approval, } \\
\text { peer influence, sexual attraction, sports }\end{array}$ \\
\hline McKillop 1987 [36] & Not specified & Not specified & Not specified & $\begin{array}{l}\text { Aggression, injury prevention/recovery, muscle, } \\
\text { sports }\end{array}$ \\
\hline Midgley 1999 [37] & Not specified & Not specified & Not specified & $\begin{array}{l}\text { Appearance, injury prevention/recovery, } \\
\text { psychological well-being, muscle, peer } \\
\text { influence, sports, sexual attraction }\end{array}$ \\
\hline Nøkleby 2013 [38] & Not specified & $\begin{array}{l}\text { Other drug use, } \\
\text { sports/exercise }\end{array}$ & Friend & $\begin{array}{l}\text { Appearance, muscle, psychological well-being, } \\
\text { sports }\end{array}$ \\
\hline O'Sullivan 2000 [39] & Not specified & Not specified & $\begin{array}{l}\text { Friends, gym dealers, } \\
\text { medical practitioners }\end{array}$ & Appearance, muscle \\
\hline Olrich 1999 [40] & 23 years & Bodybuilding & Not specified & $\begin{array}{l}\text { Appearance, curiosity, occupational, peer influence, } \\
\text { psychological well-being, social/sexual attraction, } \\
\text { sports, sport/social norm }\end{array}$ \\
\hline Pappa 2012 [41] & Not specified & Athletics & Not specified & $\begin{array}{l}\text { Appearance, concentration, curiosity, muscle, } \\
\text { social influence, sports, sport norm }\end{array}$ \\
\hline Petrocelli 2008 [42] & Not specified & $\begin{array}{l}\text { Long-term exposure } \\
\text { to muscle magazines, } \\
\text { negative body image, } \\
\text { weight training }\end{array}$ & $\begin{array}{l}\text { Dealer, friend, external } \\
\text { internet, gym dealer }\end{array}$ & $\begin{array}{l}\text { appearance, confidence, muscle, } \\
\text { psychological well-being, sexual attraction }\end{array}$ \\
\hline Pope 1990 [43] & 30 years & Weightlifting & Not specified & Not specified \\
\hline Pope 1990 [43] & 21 years & Weightlifting & Not specified & Sports \\
\hline Pope 1990 [43] & 20 years & Weightlifting & Not specified & Sports \\
\hline Pope 1993 [44] & 19 years & $\begin{array}{l}\text { Anorexia nervosa, } \\
\text { negative body image, } \\
\text { psychological } \\
\text { disorders, weightlifting }\end{array}$ & Not specified & Appearance \\
\hline Pope 1993 [44] & 18 years & $\begin{array}{l}\text { Anorexia nervosa, } \\
\text { negative body image, } \\
\text { weightlifting }\end{array}$ & Not specified & Appearance \\
\hline Pope 1993 [44] & 24 years & $\begin{array}{l}\text { Anorexia nervosa, } \\
\text { negative body image, } \\
\text { weightlifting }\end{array}$ & Not specified & Appearance \\
\hline Pope 1996 [45] & 14 years & $\begin{array}{l}\text { Psychological } \\
\text { disorders, weightlifting }\end{array}$ & Not specified & Appearance, confidence, muscle, psychological \\
\hline Rashid 2000 [14] & 38 years & $\begin{array}{l}\text { Psychological } \\
\text { disorders, other drug } \\
\text { use, troubled } \\
\text { background }\end{array}$ & Not specified & Appearance, confidence, muscle, psychological \\
\hline Schwingel 2012 [46] & Not specified & Not specified & Friends, illicit market & Appearance, muscle, occupational, sport \\
\hline Scull 2013 [47] & 18 years & Male stripping & Not specified & Appearance, muscle, occupational \\
\hline Skårberg 2008 [49] & 20 years & $\begin{array}{l}\text { Troubled background, } \\
\text { weight training }\end{array}$ & Friend & Appearance, muscle \\
\hline Skårberg 2008 [49] & 21 years & & Friend & Muscle \\
\hline
\end{tabular}


Table 2 Characteristics of qualitative studies presenting data on AAS use initiation (Continued)

\begin{tabular}{|c|c|c|c|c|}
\hline & & $\begin{array}{l}\text { Troubled background, } \\
\text { weight training }\end{array}$ & & \\
\hline Skårberg 2008 [49] & 16 years & $\begin{array}{l}\text { Irritability, troubled } \\
\text { background, weight } \\
\text { training }\end{array}$ & Not specified & Curiosity, muscle \\
\hline Skårberg 2008 [49] & 20 years & $\begin{array}{l}\text { Bodybuilding, other } \\
\text { sports }\end{array}$ & Not specified & Appearance, sports, sport norm \\
\hline Skårberg 2008 [49] & 20 years & $\begin{array}{l}\text { Bodybuilding, } \\
\text { troubled background, }\end{array}$ & Not specified & Sports, sport norm \\
\hline Skårberg 2008 [49] & 21 years & $\begin{array}{l}\text { Other sports, troubled } \\
\text { background, weight } \\
\text { training }\end{array}$ & Intimate partner & Appearance, muscle \\
\hline $\begin{array}{l}\text { Skårberg } 2009 \text { [50] } \\
\text { and } 2007 \text { [48] }\end{array}$ & 15 to 28 years & Troubled background & Not specified & Appearance, muscle, sports \\
\hline Tallon 2007 [11] & 18 to 43 years & $\begin{array}{l}\text { Weight training, other } \\
\text { sports }\end{array}$ & Friends/training partners & $\begin{array}{l}\text { Appearance, confidence, injury/illness } \\
\text { prevention, muscle, psychological, } \\
\text { sexual attraction }\end{array}$ \\
\hline Todd 1987 [51] & Not specified & Powerlifting & Dealer & Sports, sport norm \\
\hline Vassalo 2010 [52] & Not specified & Football & Not specified & Sports scholarship \\
\hline Walker 2011 [10] & 20 to 30 years $^{\dagger}$ & Not specified & Gym dealer & Appearance, muscle \\
\hline $\begin{array}{l}\text { Wilson-Fearon } \\
1999 \text { [53] }\end{array}$ & Not specified & Bodybuilding & Not specified & Sports \\
\hline
\end{tabular}

${ }^{\dagger}$ Majority.

Use of other drugs also emerged as a feature of AAS users prior to the initiation of AAS use [7,14,27,28,32,38]. Nøkleby and Skårderud [38] highlighted drug use networks as well as addiction clinics as major gateways for the initiation of use. In their study, Kristian commented:

I have always been offered steroids at other places as well, but it never came to anything. But when I got here [addiction clinic] it (steroids) fell right in my lap. And it was the same the last place I was in treatment. It (steroids) fell right in my lap, and that made it easy to accept (p. 495).

It also emerged that many AAS users understood the debilitating consequences of AAS but nevertheless went ahead to initiate use $[10,32,34,40]$. In Maycock and Howat's study [34]:

Prior to initiating [AAS] use all of the men interviewed undertook information searches. These included talking to friends, gym trainers and instructors, anabolic steroid users and dealers, reading magazines, underground anabolic steroid manuals and medical journals and occasionally talking to medical practitioners (p. 320).

\section{Sources of first AAS}

Studies specified several sources of users' first AAS: the illicit market (dealers, mail order, internet etc.), coaches or trainers, clinicians or health workers (doctors, pharmacists, and veterinarians), friends or teammates, gym employees, intimate partners, and relatives (See Table 5).

The illicit market emerged as a major source of AAS during the initiation of AAS use $[8,10,12,19,21,23,24,32$, $34,35,39,42,46,51]$. The immediate social networks of respondents such as intimate partners, relatives, as well as friends or teammates also emerged as important sources of AAS [6,11,21,23-25,28,32,38,39,42,46,49] during the initiation of AAS use.

In addition, training associates such as coaches or trainers and gym employees emerged as a source of AAS during the initiation of AAS use [15,16,19,22,24,49,55,56]. Clinicians or health workers such as doctors, pharmacists, and veterinarians also came up as sources of AAS during the initiation of AAS use [23,24,32,39].

In a 1990 study of 72 current and former users [32], the sources of AAS were: friends/teammates $(n=41)$, pharmacists $(n=22)$, dealers $(n=17)$, veterinarians $(n=$ $10)$, gym employees $(n=8)$, doctors $(n=3)$, and coach/ team doctor $(n=1)$. Moreover, in a 1997 study [24], the sources of AAS were: friends $(n=64)$, doctors $(n=42)$, dealers $(n=41)$, pharmacists $(n=18)$, gym employees $(n=14)$, coaches/trainers $(n=14)$, veterinarians $(n=11)$, relatives $(n=6)$, mail order $(n=4)$, and other $(n=4)$. It is however worthy of note that in the most recent qualitative studies presenting sources of AAS $[6,10,21,38,46]$, the only sources of AAS were the illicit market, relatives, and friends. 


\section{Motives/drives for initiating AAS use}

Motives for initiating AAS use were for: aggression, enhanced appearance, securing sports scholarships, enhanced muscle or strength, occupational (non-sporting) activities, personal security, psychological well-being or satisfaction, physiological recovery or injury prevention, sexual attraction, and for sporting or competitive activities. Other drives were trainers' approval, curiosity, family influence, use by famous athletes portrayed in the media, peer influence, and use of AAS as a sport or social norm (See Table 6).

Of the above motives and drives, initiation of AAS use for enhanced appearance or body image, muscle or strength, and sports or athletic performance were most prominent in the literature. Indeed, in a study of Australian AAS users [24], the most paramount motives for the initiation of AAS use were improved appearance (46\%), increase in size (33\%), increase in strength (7\%), and improved sporting performance (6\%). Case 04 of Fudala et al.'s study [25] also tells the story of a 22-year-old male who initiated AAS use at the age of 17 "in order to increase his size and power for football" and consecutively increased his AAS consumption "in order to compete in bodybuilding events". Paula, a 39-year-old affirms the relationship between her AAS use and sports participation in Grogan et al.'s study [26] with the confession "I will stop [using steroids] when I stop competing yeah" (p. 853). Similarly, others initiated AAS use for physiological recovery or injury prevention $[9,11,12,20,21,23,24,28,32,33,36,37]$.

Related to enhanced sports performance, enhanced occupational functioning also emerged as motive for the initiation of AAS use $[6,26,34,35,40,46,47]$. In support of this motive, Matt, a 33-year-old male stripper commented in Scull's study [47]: "All the guys [male strippers] take steroids, you know?...See, you won't last long in this industry if you don't use steroids. They all do steroids" (p. 567). Improved occupational functioning was again highlighted in Maycock and Howat's study [35]:

For the doormen and security workers, it was about projecting physical competence; for the power lifters, it was about projecting the image of brute strength; for the sex workers or gay men using for body image reasons, it was about the presentation of a natural healthy look. For bodybuilders, it was about projecting their muscles, size and shape (p. 861).

Sexual attraction or attractiveness also emerged as an important motive for the initiation of AAS use [11,32,40,42]. This is highlighted by Kusserow's [32] finding that 18\% of AAS users initiated use in order to "be more successful with the opposite sex" (p. 7). In addition, Petrocelli et al. [42] indicated that AAS use: increased and enhanced [users'] confidence and love life, as they claimed having a defined, muscular physique allowed them to meet and have sexual relations with more partners (p. 1194).

Social pressure in the form of media influence, peer influence, and sport or social norms also emerged as an important drive for the initiation of AAS use. Related to this, Petrocelli et al. [42] found long-term exposure to muscle magazines as a feature of AAS users prior to initiation of AAS use. In addition, Joe a 29-year-old male commented: "I came from a solid family that stressed competition and giving it $110 \%$. So when I didn't see the results in the gym, I went to steroids" [22, p. 10]. In Grogan et al.'s study [26], John, a 25-year-old indicated:

The more I trained, the more magazines I looked at, the bigger I wanted to be. ... and there was an ITV programme [about body builders] and when I watched these people it made me feel really depressed. I didn't look as good as them. And it had a massive effect on my decision to take steroids. In fact it was probably one of the biggest reasons why I did take them seeing other people bigger than me (p. 853).

There is however contrary evidence of the influence of media on AAS use. In Walker and Joubert's study [10], $66 \%$ of respondents stated that the media had no influence on their desire to use AAS although these respondents believed that most muscular men portrayed in the media use AAS.

Moreover, psychological well-being emerged as an important motive for the initiation of AAS use [7,10,11, $14,20,21,25,31,37,38,40,42,45]$. Specific psychological motives for initiating use included boosting self-esteem, confidence, concentration, and overcoming psychological disorders such as depression.

It is important to note however that motives for AAS use may change with time. For instance, in an Australian study [24], 46\% of users indicated that they initiated use in order to improve their appearance. However, only $35 \%$ of these respondents mentioned improved appearance as motive for their most recent use indicating motive change in some users after initiation. Disparities were also discovered for other motives (p. 37). A security worker also elucidated motive change in a recent study by Kimergård [6]:

At this moment in time, I'm not looking to get any bigger as a bodybuilder for example. I like to increase my strength, and now it's more for conditioning... My next cycle, I'll be doing a 'cutting' cycle, I'll be dieting and getting down to a reasonable healthy weight (p. 3). 
Table 3 Qualitative studies presenting age(s) of AAS use initiation

\begin{tabular}{|c|c|}
\hline Age(s) of initiation & Studies (first author, reference) \\
\hline 14 years & Copeland [23] and Peters [24]; Kusserow [32]; Pope [45]; Tallon [11] \\
\hline 15 years & Copeland [23] and Peters [24]; Grogan [26]; Kusserow [32]; Skårberg [48,50]; Tallon [11] \\
\hline 16 years & $\begin{array}{l}\text { Annitto [19]; Copeland [23] and Peters [24]; Grogan [26]; Korkia [12]; Skårberg [49]; } \\
\text { Kimergård [8]; Joubert [7]; Tallon [11] }\end{array}$ \\
\hline 17 years & Copeland [23] and Peters [24]; Fudala [25]; Kusserow [32]; Tallon [11] \\
\hline 18 years & Copeland [23] and Peters [24]; Grogan [26]; Korkia [12]; Kusserow [32]; Pope [44]; Scull [47]; Joubert [7]; Tallon [1 1] \\
\hline 19 years & Boyadjiev [22]; Copeland [23] and Peters [24]; Grogan [26]; Korkia [13]; Pope [44]; Joubert [7]; Tallon [11] \\
\hline 20 years & Copeland [23] and Peters [24]; Cornford [9]; Grogan [26]; Pope [43]; Skårberg [49]; Tallon [11] \\
\hline 21 years & $\begin{array}{l}\text { Copeland [23] and Peters [24]; Cornford [9]; Fudala [25]; Grogan [26]; Katz [29]; } \\
\text { Pope [43]; Skårberg [49]; Tallon [11] }\end{array}$ \\
\hline 22 years & Copeland [23] and Peters [24]; Cornford [9]; Hegazy [28] \\
\hline 23 years & Copeland [23] and Peters [24]; Cornford [9]; Grogan [26]; Korkia [13]; Olrich [40]; Tallon [11] \\
\hline 24 years & $\begin{array}{l}\text { Copeland [23] and Peters [24]; Cornford [9]; Fudala [25]; Malone [33]; Maycock [34,35]; } \\
\text { Pope [44]; Joubert [7]; Tallon [11] }\end{array}$ \\
\hline 25 years & Copeland [23] and Peters [24]; Cornford [9]; Maycock [34,35]; Tallon [11] \\
\hline 26 years & Copeland [23] and Peters [24]; Cornford [9]; Fudala [25]; Tallon [11] \\
\hline 27 years & Copeland [23] and Peters [24]; Cornford [9]; Fudala [25]; Tallon [11] \\
\hline 28 years & Copeland [23] and Peters [24]; Cornford [9]; Skårberg [48,50]; Tallon [1 1] \\
\hline 29 years & Copeland [23] and Peters [24]; Cornford [9]; Grogan [26]; Tallon [11] \\
\hline 30 years & Copeland [23] and Peters [24]; Cornford [9]; Pope [43]; Tallon [11] \\
\hline 31 to 54 years & Copeland [23] and Peters [24]; Cornford [9]; Korkia [12]; Rashid [14]; Tallon [11] \\
\hline Not specified & $\begin{array}{l}\text { Bardick [20]; Bilard [21]; Fudala [25]; Gruber [27]; Joubert [7]; Katz [29]; Khorrami [30]; Kimergård [6,8]; } \\
\text { Klötz [31]; Korkia [12,13]; Kusserow [32]; Maycock [34,35]; McKillop [36]; Midgley [37]; Nøkleby [38]; } \\
\text { O'Sullivan [39]; Olrich [40]; Petrocelli [42]; Schwingel [46]; Scull [47]; Skårberg [48,50]; Tallon [11]; } \\
\text { Todd [51]; Vassalo [52]; Walker [10]; Wilson-Fearon [53] }\end{array}$ \\
\hline
\end{tabular}

Not specified: Authors did not present age(s) of initiation for some or all participants.

Table 4 Qualitative studies presenting AAS users' history prior to use

\begin{tabular}{ll}
\hline History & Studies (first author, reference) \\
\hline Anorexia and reverse anorexia & Fudala [25]; Pope [44] \\
$\begin{array}{l}\text { Complacent trainer(s) } \\
\text { Long-term exposure to muscle }\end{array}$ & Petrocelli [42] \\
magazines & \\
Low self-efficacy & Fudala [25]; Joubert [7] \\
Male sex work & Scull [47] \\
Negative body image & Fudala [25]; Khorrami [30]; Kusserow [32]; Maycock [34,35]; Petrocelli [42]; Pope [44]; Walker [10]; Joubert [7] \\
Other drug(s) use & Gruber [27]; Hegazy [28]; Joubert [7]; Kusserow [32]; Nøkleby [38]; Rashid [14] \\
Other sports (athletics, cycling, hockey, & Bardick [20]; Boyadjiev [22]; Fudala [25]; Joubert [7]; Khorrami [30]; Kusserow [32]; Nøkleby [38]; Pappa [41]; \\
football etc.) & Skårberg [49]; Tallon [11]; Vassalo [52] \\
Poor self-esteem & Fudala [25]; Walker [10]; Joubert [7] \\
Power sports (bodybuilding, & Annitto [19]; Bardick [20]; Bilard [21]; Boyadjiev [22]; Fudala [25]; Grogan [26]; Joubert [7]; Katz [29]; \\
powerlifting, weightlifting) & Khorrami [30]; Kimergård [8]; Korkia [12]; Kusserow [32]; Malone [33]; Maycock [34,35]; Olrich [40]; \\
& Petrocelli [42]; Pope [43]; Pope [44,45]; Skårberg [49]; Tallon [11]; Todd [51]; Wilson-Fearon [53] \\
Psychological disorder & Fudala [25]; Gruber [27]; Hegazy [28]; Pope [44,45]; Rashid [14] \\
Troubled background (bullying, & Fudala [25]; Gruber [27]; Hegazy [28]; Rashid [14]; Skårberg [48-50]; Joubert [7] \\
\hline divorce, rape etc.) & \\
\hline
\end{tabular}


Table 5 Qualitative studies presenting AAS users' first sources of AAS

\begin{tabular}{ll}
\hline Source & Studies (first author, reference) \\
\hline Coach/trainer & Copeland [23] and Peters [24]; Gruber [27]; Korkia [12,13]; Kusserow [32] \\
Doctor & Copeland [23] and Peters [24]; Korkia [12]; Kusserow [32]; O'Sullivan [39] \\
Friend/teammate & Bilard [21]; Copeland [23] and Peters [24]; Fudala [25]; Hegazy [28]; Kimergård [6]; Korkia [12]; \\
& Kusserow [32]; Nøkleby [38]; O'Sullivan [39]; Petrocelli [42]; Schwingel [46]; Skårberg [49]; Tallon [11] \\
Gym employee & Copeland [23] and Peters [24]; Khorrami [30]; Korkia [12,13]; Kusserow [32]; Walker [10] \\
Illicit market (dealers, internet) & Annitto [19]; Bilard [21]; Copeland [23] and Peters [24]; Kimergård [8]; Korkia [12]; Kusserow [32]; \\
Intimate partner & Maycock [34,35]; O'Sullivan [39]; Petrocelli [42]; Schwingel [46]; Todd [51]; Walker [10] \\
Pharmacist & Korkia [13]; Skårberg [49] \\
Relative & Copeland [23] and Peters [24]; Kusserow [32] \\
Veterinarian & Bilard [21]; Copeland [23] and Peters [24]; Fudala [25] \\
\hline
\end{tabular}

\section{Implications for research}

The results of our study have important implications for future investigations. First, unnecessary replication of qualitative research may be avoided when systematic reviews and qualitative syntheses are conducted prior to the execution of new qualitative research. In addition, all studies were conducted in Western countries. This is problematic as there is evidence that nonmedical AAS use represents a global public health problem [1]. Thus, future studies must as well endeavour to investigate the experiences of AAS users in non-Western countries.

Our findings also reveal a relative paucity of qualitative investigations on the influence of backgrounds of anorexia nervosa, complacent trainers, use of other appearance and performance enhancing drugs and methods, long-term exposure to media images of muscular persons, low selfesteem and self-efficacy, and male sex work on the initiation of AAS use. Moreover, scant qualitative studies have

Table 6 Qualitative studies presenting AAS users' motives/drives for initiating AAS use

\begin{tabular}{|c|c|}
\hline Motive/drive & Studies (first author, reference) \\
\hline Aggression & Klötz [31]; Kusserow [32]; Maycock [34,35]; Mckillop [36] \\
\hline Appearance/body image & $\begin{array}{l}\text { Annitto [19]; Bardick [20]; Bilard [21]; Copeland [23] and Peters [24]; Fudala [25]; Grogan [26]; Gruber [27]; } \\
\text { Hegazy [28]; Khorrami [30]; Kimergård [6,8]; Klötz [31]; Kusserow [32]; Malone [33]; Maycock [34,35]; Midgley } \\
\text { [37]; Nøkleby [38]; O'Sullivan [39]; Olrich [40]; Pappa [41]; Petrocelli [42]; Pope [44,45]; Rashid [14]; Schwingel } \\
\text { [46]; Scull [47]; Skårberg [48-50]; Tallon [11]; Walker [10] }\end{array}$ \\
\hline Coach's/trainer's approval/influence & Kusserow [32]; Maycock [34,35] \\
\hline Curiosity & Olrich [40]; Pappa [41]; Skårberg [49] \\
\hline Family influence & Khorrami [30]; Kusserow [32]; Joubert [7] \\
\hline Media & Bardick [20]; Grogan [26]; Kusserow [32]; Pappa [41]; Walker [10] \\
\hline Muscle/strength & $\begin{array}{l}\text { Bilard [21]; Copeland [23] and Peters [24]; Cornford [9]; Fudala [25]; Gruber [27]; Hegazy [28]; Joubert [7]; } \\
\text { Khorrami [30]; Kimergård [6]; Klötz [31]; Korkia [12,13]; Malone [33]; McKillop [36]; Midgley [37]; Nøkleby [38]; } \\
\text { O'Sullivan [39]; Pappa [41]; Petrocelli [42]; Pope [45]; Rashid [14]; Schwingel [46]; Scull [47]; Skårberg [49]; } \\
\text { Skårberg [48,50]; Tallon [11]; Walker [10] }\end{array}$ \\
\hline Occupational (non-sporting) & Grogan [26]; Kimergård [6]; Maycock [35]; Olrich [40]; Schwingel [46]; Scull [47] \\
\hline Peer influence & Joubert [7]; Kusserow [32]; Maycock [34,35]; Midgley [37]; Olrich [40] \\
\hline Personal security & Bardick [20]; Cornford [9]; Gruber [27]; Joubert [7] \\
\hline $\begin{array}{l}\text { Physiological recovery/injury } \\
\text { prevention }\end{array}$ & $\begin{array}{l}\text { Bardick [20]; Bilard [21]; Copeland [23] and Peters [24]; Cornford [9]; Hegazy [28]; Korkia [12]; Kusserow [32]; } \\
\text { Malone [33]; McKillop [36]; Midgley [37]; Tallon [11] }\end{array}$ \\
\hline $\begin{array}{l}\text { Psychological (well-being, self-esteem, } \\
\text { self-efficacy, concentration, confidence) }\end{array}$ & $\begin{array}{l}\text { Bardick [20]; Bilard [21]; Fudala [25]; Joubert [7]; Klötz [31]; Midgley [37]; Nøkleby [38]; Olrich [40]; Petrocelli } \\
\text { [42]; Pope [45]; Rashid [14]; Tallon [11]; Walker [10] }\end{array}$ \\
\hline Sexual attraction/attractiveness & Kusserow [32]; Olrich [40]; Petrocelli [42]; Tallon [11] \\
\hline Sport/social norm & Bilard [21]; Grogan [26]; Klötz [31]; Malone [33]; Olrich [40]; Pappa [41]; Skårberg [49]; Todd [51]; Kimergård [8] \\
\hline Sports & $\begin{array}{l}\text { Bardick [20]; Bilard [21]; Boyadjiev [22]; Copeland [23] and Peters [24]; Fudala [25]; Grogan [26]; Joubert [7]; } \\
\text { Katz [29]; Khorrami [30]; Klötz [31]; Korkia [12,13]; Kimergård [6]; Kusserow [32]; Malone [33]; Maycock [34,35]; } \\
\text { McKillop [36]; Midgley [37]; Nøkleby [38]; Olrich [40]; Pappa [41]; Pope [43]; Schwingel [46]; Skårberg [48-50]; } \\
\text { Todd [51]; Wilson-Fearon [53] }\end{array}$ \\
\hline Sports scholarship & Kusserow [32]; Vassalo [52] \\
\hline
\end{tabular}


examined the influence of motives and drives such as securing sports scholarships, coaches' or trainers' approval, the search for confidence, curiosity, the influence of famous athletes, family influence, and personal security on the initiation of AAS use. Thus, future studies should examine these topics.

\section{Implications for policy and practice}

Arguably, our findings represent an important basis for policymaking and planning. First, with evidence from the present study that most AAS users initiate use under 30 years, AAS use interventions should focus primarily on adolescents and young adults. Thus preventive interventions should be tailored mainly for these age cohorts. In addition, with evidence from our study that negative body image, psychological disorders, and sports participation (particularly in power sports) precede initiation of AAS use for most persons, AAS use interventions must target persons demonstrating these characteristics as well as focus on relevant environments.

Moreover, AAS use interventions must be targeted at individuals with: eating disorders, low self-esteem and self-efficacy, 'doping-complacent' trainers, long-term exposure to media images of muscular persons, troubled backgrounds, drug use histories and milieus, and psychological disorders. AAS use interventions should also be aimed at athletes especially power sportspeople, doormen and security workers, male sex workers, and gay men as these groups emerged as popular AAS users in this qualitative metasynthesis.

Again, it is worrying that although some AAS users appreciated the debilitating consequences of AAS, they nevertheless went ahead to initiate use $[6,10,32,34,40]$. We also found that sources of first AAS were mainly users' immediate social networks and the illicit market. Furthermore, it is worthy of note that in the most recent qualitative studies presenting sources of AAS $[6,10,21,38,46]$, the only sources were the illicit market, relatives, and friends. This is perhaps attributable to the increasing illegalization of AAS use since the 1990s [1]. Nevertheless, with the proliferation of both legal and illegal substances on the illicit market and the internet, as well as the expectedly 'drug-clean' environments of addiction clinics [38], better ways of dealing with the global public health problem of AAS use will need to be found.

\section{Strengths and weaknesses}

The present study has several strengths. To our knowledge, it is the first-ever systematic review and synthesis of qualitative studies on AAS use initiation. The systematic and advanced strategy for identifying, reporting, and synthesizing qualitative studies, the 'global' and comprehensive nature of the present study, and the inclusion of a large number of both peer-reviewed and grey literature are also notable assets.

Despite the aforementioned strengths of the present study, some limitations ought to be noted when interpreting our results. First, we restricted our analysis to English language literature. Though this is not an uncommon practice for systematic reviews [57], it is possible that the exclusion of non-English language literature influenced our results. However, it must be noted that Moher et al. [57] found no evidence of biased results with the exclusion of non-English studies. Nevertheless, it is worth pointing out again that our themes and results were robust in the sensitivity analysis. Furthermore, it is plausible that the case studies included in the present study were reported due to their 'unusual' or 'exceptional' nature. Thus, these cases may not be representative of the typical AAS user.

\section{Conclusions}

Arguably, our findings represent an important basis for AAS use interventions. Findings from the present study denote the importance of psychological and social factors in the initiation of AAS use. Our findings also complement available evidence from quantitative studies on the initiation of AAS use. There is the need for improved ways of dealing with the global problem of AAS use with the increased availability of both legal and illegal substances on the illicit market and the internet.

\section{Competing interests}

The authors declare that they have no competing interests.

\section{Authors' contributions}

DS led the conception and design of the study, the literature search, analysis, writing and revision of the manuscript. CSA and SP contributed to the writing and revision of the manuscript. All authors read and approved the final manuscript.

\section{Authors' information}

DS is a PhD research fellow at the Department of Psychosocial Science, University of Bergen, Norway. He conducts research on image and performance enhancing drugs and methods with special focus on anabolic-androgenic steroids. He also works on other drug and behavioural addictions. CSA is a postdoctoral research fellow at the Department of Psychosocial Science, University of Bergen, Norway, and a clinical psychologist at the Bergen Clinics Foundation, Norway. She conducts research in the area of work, industrial and organizational psychology, as well as drug and behavioural addictions. SP is a professor of psychology at the Department of Psychosocial Science, University of Bergen, Norway, and a senior researcher at the Norwegian Competence Centre for Sleep Disorders. He conducts research on sleep and sleep disorders as well as drug and behavioural addictions.

\section{Acknowledgements}

We are grateful to Jim McVeigh for his contribution to the literature search process. We thank Philomena Antwi for reviewing studies included in the narrative synthesis.

\section{Author details}

${ }^{1}$ Department of Psychosocial Science, University of Bergen, Christiesgate 12, 5015 Bergen, Norway. ${ }^{2}$ The Competence Centre, Bergen Clinics Foundation, Bergen, Norway. 
Received: 7 May 2014 Accepted: 26 June 2014

Published: 2 July 2014

\section{References}

1. Sagoe D, Molde H, Andreassen CS, Torsheim T, Pallesen S: The global epidemiology of anabolic-androgenic steroid use: a meta-analysis and meta-regression analysis. Ann Epidemiol 2014, 24:383-398.

2. Kanayama G, Boynes M, Hudson JI, Field AE, Pope HG Jr: Anabolic steroid abuse among teenage girls: an illusory problem? Drug Alcohol Depend 2007, 88:156-162.

3. Lawlor DA, Frankel S, Shaw M, Ebrahim S, Smith GD: Smoking and ill health: does lay epidemiology explain the failure of smoking cessation programs among deprived populations? Am J Public Health 2003, 93:266-270.

4. Marshall IJ, Wolfe CD, McKevitt C: Lay perspectives on hypertension and drug adherence: systematic review of qualitative research. BMJ 2012, 345:e3953.

5. Popay J, Roberts R, Sowden A, Petticrew M, Arai L, Rodgers M, Britten N, Roen K, Duffy S: Guidance on the conduct of narrative synthesis in systematic reviews. A product from the ESRC methods programme. York: University of York; 2006.

6. Kimergård A: A qualitative study of anabolic steroid use amongst gym users in the United Kingdom: motives, beliefs and experiences. J Subst Use 2014, doi:10.3109/14659891.2014.911977 [Epub ahead of print].

7. Joubert HE: What is the role of shame for male anabolic androgenic steroid users? Leicester, UK: University of Leicester; 2014. Retrieved May 2014 from https://lra.le.ac.uk/bitstream/2381/28833/1/2014JoubertHEPsyD.pdf.

8. Kimergård A, McVeigh J: Environments, risk and health harms: a qualitative investigation into the illicit use of anabolic steroids among people using harm reduction services in the UK. BMJ Open 2014, 4:e005275. doi:10.1136/ bmjopen-2014-005275.

9. Cornford CS, Kean J, Nash A: Anabolic-androgenic steroids and heroin use: a qualitative study exploring the connection. Int J Drug Policy 2014. http://dx.doi.org/10.1016/j.drugpo.2014.06.002 [Epub ahead of print].

10. Walker DM, Joubert HE: Attitudes of injecting male anabolic androgenic steroid users to media influence, health messages and gender constructs. Drugs Alcohol Today 2011, 11:56-70.

11. Tallon V: An Exploratory Investigation of Anabolic-Androgenic Steroid use in Lanarkshire. Paisley: University of Paisley; 2007. Retrieved June 2014 from http://www.ibrarian.net/navon/page.jsp?paperid=10804537\&searchTerm= east+kilbride.

12. Korkia P, Stimson G: Anabolic Steroid use in Great Britain: An Exploratory Investigation. Final Report to the Department of Health for England, Scotland and Wales. London: The Centre for Research on Drugs and Health Behaviour; 1993.

13. Korkia P, Lenehan P, McVeigh J: Non-medical use of androgens among women. J Perform Enhanc Drugs 1996, 1:71-76.

14. Rashid W: Testosterone abuse and affective disorders. J Subst Abuse Treat 2000, 18:179-184.

15. Shaw R, Booth A, Sutton A, Miller T, Smith JA, Young B, Jones DR, DixonWoods $\mathrm{M}$ : Finding qualitative research: an evaluation of search strategies. BMC Med Res Methodol 2004, 4:5.

16. Moher D, Liberati A, Tetzlaff J, Altman DG, The PRISMA Group: Preferred reporting items for systematic reviews and meta-analyses: the PRISMA statement. PLoS Med 2009, 6:e1000097.

17. Smith JA, Harré R, Van Langenhove L: Idiography and the case study. In Rethinking Psychology. Edited by Smith JA, Harre R, Van Langenhove L. London: Sage; 1995:57-69.

18. Smith JA, Osborn M: Interpretative phenomenological analysis. In Qualitative Psychology: a Practical Guide to Methods. Edited by Smith JA. London: Sage; 2008:53-80.

19. Annito WR, Layman WA: Anabolic steroids and acute schizophrenic episode. J Clin Psychiatry 1980, 41:143-144.

20. Bardick $A D$, Bernes $K B$, Nixon $G:$ In pursuit of physical perfection: weight lifting and steroid use in men. J Excellence 2006, 11:135-145.

21. Bilard J, Ninot G, Hauw D: Motives for illicit use of doping substances among athletes calling a national antidoping phone-help service: an exploratory study. Subst Use Misuse 2011, 46:359-367.

22. Boyadjiev N, Georgieva K, Massaldjieva R, Gueorguiev S: Reversible hypogonadism and azoospermia as a result of anabolic-androgenic steroid use in a bodybuilder with personality disorder. A case report. J Sports Med Phys Fitness 2000, 40:271-274.
23. Copeland J, Peters R, Dillon P: Anabolic-androgenic steroid use disorders among a sample of Australian competitive and recreational users. Drug Alcohol Depend 2000, 60:91-96.

24. Peters R, Copeland J, Dillon P, Beel A: Patterns and Correlates of AnabolicAndrogenic Steroid use. Technical Report No 48. Sydney: National Drug and Alcohol Research Centre; 1997.

25. Fudala PJ, Weinrieb RM, Calarco JS, Kampman KM, Boardman C: An evaluation of anabolic-androgenic steroid abusers over a period of 1 year: seven case studies. Ann Clin Psychiatry 2003, 15:121-130.

26. Grogan S, Shepherd S, Evans R, Wright S, Hunter G: Experiences of anabolic steroid use: in-depth interviews with men and women body builders. J Health Psychol 2006, 11:845-856.

27. Gruber AJ, Pope HG Jr: Compulsive weight lifting and anabolic drug abuse among women rape victims. Compr Psychiatry 1999, 40:273-277.

28. Hegazy B, Sanda C: A 28-year-old man with depression, PTSD, and anabolic-androgenic steroid and amphetamine use. Psychiatr Ann 2013, 43:408-411.

29. Katz DL, Pope HG Jr: Anabolic-androgenic steroid-induced mental status changes. NIDA Res Monogr 1990, 102:215-223.

30. Khorrami S, Franklin J: The influence of competition and lack of emotional expression in perpetuating steroid abuse and dependence in male weightlifters. IJMH 2002, 1:119-133.

31. Klötz F, Petersson A, Hoffman O, Thiblin I: The significance of anabolic androgenic steroids in a Swedish prison population. Compr Psychiatry 2010, 51:312-318

32. Kusserow RP: Adolescents and Steroids: A User Perspective. Washington, DC: Office of Inspector General; 1990.

33. Malone DA Jr, Dimeff RJ, Lombardo JA, Sample RB: Psychiatric effects and psychoactive substance use in anabolic-androgenic steroid users. Clin J Sport Med 1995, 5:25-31.

34. Maycock B, Howat P: Overcoming the barriers to initiating illegal anabolic steroid use. Drug Educ Prev Policy 2005, 14:317-325.

35. Maycock B, Howat P: Social capital: implications from an investigation of illegal anabolic steroid networks. Health Educ Res 2007, 22:854-863.

36. McKillop G: Drug abuse in bodybuilders in the west of Scotland. Scott Med J 1987, 32:39-41.

37. Midgley SJ, Heather N, Davies JB: Dependence-producing potential of anabolic-androgenic steroids. Addict Res 1999, 7:539-550.

38. Nøkleby H, Skårderud F: Body practices among male drug abusers. Meanings of workout and use of doping agents in a drug treatment setting. Int J Ment Health Addict 2013, 11:490-502.

39. O'Sullivan AJ, Kennedy MC, Casey JH, Day RO, Corrigan B, Wodak AD: Anabolic- androgenic steroids: medical assessment of present, past and potential users. Med J Aust 2000, 173:323-327.

40. Olrich TW, Ewing ME: Life on steroids: bodybuilders describe their perceptions of the anabolic-androgenic steroid use period. Sport Psychol 1999, 13:299-312.

41. Pappa E, Kennedy E: "It was my thought ... he made it a reality": normalization and responsibility in athletes' accounts of performance enhancing drug use. Int Rev Sociol Sport 2012, 48:277-294.

42. Petrocelli M, Oberweis T, Petrocelli J: Getting huge, getting ripped: a qualitative exploration of recreational steroid use. J Drug Issues 2008, 38:1187-1206.

43. Pope HG Jr, Katz DL: Homicide and near-homicide by anabolic steroid users. J Clin Psychiatry 1990, 51:28-31.

44. Pope HG Jr, Katz DL, Hudson Jl: Anorexia nervosa and "reverse anorexia" among 108 male bodybuilders. Compr Psychiatry 1993, 34:406-409.

45. Pope HG Jr, Kouri EM, Powell KF, Campbell C, Katz DL: Anabolic-androgenic steroid use among 133 prisoners. Compr Psychiatry 1996, 37:322-327.

46. Schwingel PA, Cotrim HP, dos Santos CR Jr, Salles BCR, de Almeida CER, Zoppi CC: Non-medical anabolic-androgenic steroid consumption and hepatitis $B$ and $C$ virus infection in regular strength training practitioners. J Pharm Pharmacol 2012, 6:1598-1605.

47. Scull MT: Reinforcing gender roles at the male strip show: a qualitative analysis of men who dance for women (MDW). Deviant Behav 2013, 34:557-578.

48. Skårberg K, Engstrom I: Troubled social background of male anabolicandrogenic steroid abusers in treatment. Subst Abuse Treat Prev Policy 2007, 2:20.

49. Skårberg K, Nyberg F, Engström I: The development of multiple drug use among anabolic-androgenic steroid users: six subjective case reports. Subst Abuse Treat Prev Policy 2008, 3:24. 
50. Skårberg K, Nyberg F, Engström I: Multisubstance use as a feature of addiction to anabolic-androgenic steroids. Eur Addict Res 2009, 15:99-106

51. Todd T: Anabolic steroids: the gremlins of sport. J Sport Hist 1987, 14:87-107.

52. Vassallo MJ, Olrich TW: Confidence by injection: male users of anabolic steroids speak of increases in perceived confidence through anabolic steroid use. Int I Sport Exerc Psychol 2010, 8:70-80.

53. Wilson-Fearon C, Parrott AC: Multiple drug use and dietary restraint in a Mr. Universe competitor: psychobiological effects. Percept Mot Skills 1999, 88:579-580

54. IBM Corp: IBM SPSS Statistics for Windows, Version 20.0. Armonk, NY: IBM Corp; 2011.

55. Viera AJ, Garrett JM: Understanding interobserver agreement: the kappa statistic. Fam Med 2005, 37:360-363.

56. Pope HG Jr, Kanayama G, Athey A, Ryan E, Hudson JI, Baggish A: The lifetime prevalence of anabolic-androgenic steroid use and dependence in Americans: Current best estimates. Am J Addict 2013, doi:10.1111/j.15210391.2013.12118x [Epub ahead of print].

57. Moher D, Pham B, Klassen TP, Schulz KF, Berlin JA, Jadad AR, Liberati A: What contributions do languages other than English make on the results of meta-analyses? J Clin Epidemiol 2000, 53:964-972.

doi:10.1186/1747-597X-9-27

Cite this article as: Sagoe et al:: The aetiology and trajectory of anabolic-androgenic steroid use initiation: a systematic review and synthesis of qualitative research. Substance Abuse Treatment, Prevention, and Policy 2014 9:27.

\section{Submit your next manuscript to BioMed Central and take full advantage of:}

- Convenient online submission

- Thorough peer review

- No space constraints or color figure charges

- Immediate publication on acceptance

- Inclusion in PubMed, CAS, Scopus and Google Scholar

- Research which is freely available for redistribution 